\title{
Performance Optimization of Unit-Cell Reflectarray Antenna for Future 5G Communications
}

\author{
Muhammad Arslan Sheikh \\ UET Peshawar
}

\author{
Shahzada Alamgir Khan \\ ICT, Islamabad
}

\begin{abstract}
In this paper, Reflectarray antenna design operating in KaBand at $28 \mathrm{GHz}$ for the future fifth generation $(5 \mathrm{G})$ wireless communication applications is presented. This reflectarray antenna is proposed and designed based on the two different resonant elements i.e. circular loop with split rings and square loop with split rings. The characterization of each reflectarray element is further carried out in terms of reflection loss, reflection phase, $10 \%$ bandwidth and static phase range, respectively. Simulations have been performed using CST MWS simulation software based on Finite Integral Method (FIM). The $10 \%$ bandwidth, Figure of Merit (FOM) and the reflection characteristics (including reflection loss and reflection phase) have demonstrated the possibility of using the designated reflectarray antenna optimizely for $5 \mathrm{G}$ wireless communications. These antennas have a significant advantage of being light weight and cost effective as compared to the existing conventional bulky reflectors and phased arrays. Due to the remarkable performance of the proposed reflectarray antenna array, it can be considered as a good candidate for $5 \mathrm{G}$ communication applications.
\end{abstract}

\section{Keywords}

Reflectarray antenna, Finite Integral Method (FIM), Figure of Merit (FOM), Bandwidth, Gain, Fifth Generation (5G), Wireless communication, Reflection phase and Reflection loss.

\section{INTRODUCTION}

\subsection{Traditional Antennas}

Antennas can be designed to transmit and receive radio waves either in all horizontal directions equally (Omni directional antennas) or preferentially in a particular direction (Directional or High gain antennas) [1]. Traditional metallic antenna consists of metallic conductors, electrically connected (often through a transmission line) to the receiver or transmitter. An oscillating current creates a magnetic field around the antenna elements [2]. These time varying fields radiate away from the antenna into space as a moving transverse electromagnetic wave. Conversely, during reception, the oscillating electric and magnetic fields of an incoming radio wave exert force on the electrons in the antenna elements causing them to move back and forth, creating oscillating currents in the antenna.

Antennas are characterized by a number of performance measures which a user would be connected with in selecting or designing an antenna for a particular application. Two major factors associated with the design of antennas are the antenna resonant frequency or center operating frequency and the antenna bandwidth or the frequency range over which the antenna operates.

\subsection{Reflectarray Antenna}

To meet the heavy mobile data traffic in 2020, a few trends have seemed in the way to layout $5 \mathrm{G}$ networks [3]. In this work, unit-cell reflectarray antenna is exploited to target kaband for 5G wireless communication applications. The reflectarray antenna layout allows for extra directional antennas which can be electrically steerable and versatility smart over existing mechanical antennas.

\section{FEATURES OF REFLECTARRAY ANTENNA}

- A reflectarray antenna is a kind of antenna that syndicates the best features of two well-known high gains antennas i.e. (1.) Reflector Antenna and (2.) Phased Arrays.

The features like feeding techniques of Reflector Antenna and design principles of phased arrays, both are present in reflectarray antenna.

- The main feature of reflectarray antenna is its feeding technique, where the reflecting surface is illuminated spatially by a suitable located feed antenna.

- Since the number of elements on a reflectarray is typically in the hundreds or thousands, the high gain feature of reflectarray antenna becomes inherent in nature.

- The high gain property of reflectarray antenna has made it very attractive for space and satellite communication where the planar nature of the reflecting surface offers an added advantage when compared to parabolic reflectors in terms of shape and compactness.

- Reflectarray antennas are easily fixed on the surface of a structure having flexible installation, low production cost, steerable beam, integrated with a solar array and its efficiency is improved by designing a large aperture array.

\section{APPLICATIONS OF}

\section{REFLECTARRAY ANTENNA}

- The reflectarray antennas have multiple field applications like Direct Broadcast Satellite (DBS) applications, Multi Beam Antenna (MBA) applications, Earth Observatory \& Multi Frequency Radar Systems and Vehicular Systems used for Mobile Communications and Defense applications.

- The reflectarray antenna systems have simple beam forming network and low cost send/receive amplifier modules as compared to other antenna systems due to its wide-angle beam scanning capabilities where the main beam can tilt at a big angle i.e. round about $50^{\circ}$ from its broad side direction.

- As the power-divider is no longer compulsory and very slight resistive-insertion loss has been patterned in reflectarray antennas, it can achieve more than 50\% efficiency. 
- Due to the flat/simpler shape of reflectarray antenna systems, it is handy to mount the new antennas on it.

- The fabrication of reflectarray antennas is very simple and inexpensive especially when manufactured in bulk.

- The elemental-phase adjustment can be possible due to the number of series of radiating elements being used in reflectarray antenna surface. Therefore, by using PhaseSynthesis technique, the reflectarray antennas have very precise contour beam-shape.

By using simple Photo-Etching procedures, Reflectarrays have the capability to form contoured and fixed-focused beams. With the invention of control devices on the reflecting elements, Reconfigurable Beam Reflectarray antenna systems have also been developed. There are numerous applications of reflectarray which are introduced in space as well as shown in the Figure 1.
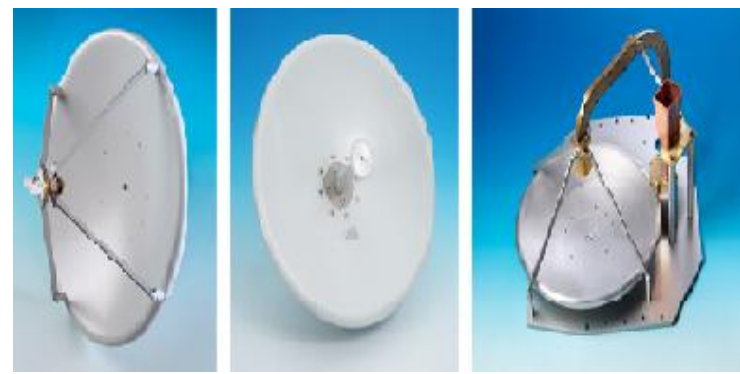

Fig 1: Reflectarray Antennas for DBS (Direct Broadcast Satellite)-Applications

\section{FEED CONFIGURATIONS OF REFLECTARRAY ANTENNA}

As the reflectarray antennas are the typical type of antennas that comprises the best features of both two well-known high gain antennas.

The feeding techniques are the possible main features of the reflector antennas. Here with the help of a suitable located feed antenna, the reflecting surface is illuminated spatially as shown in figure 2 .

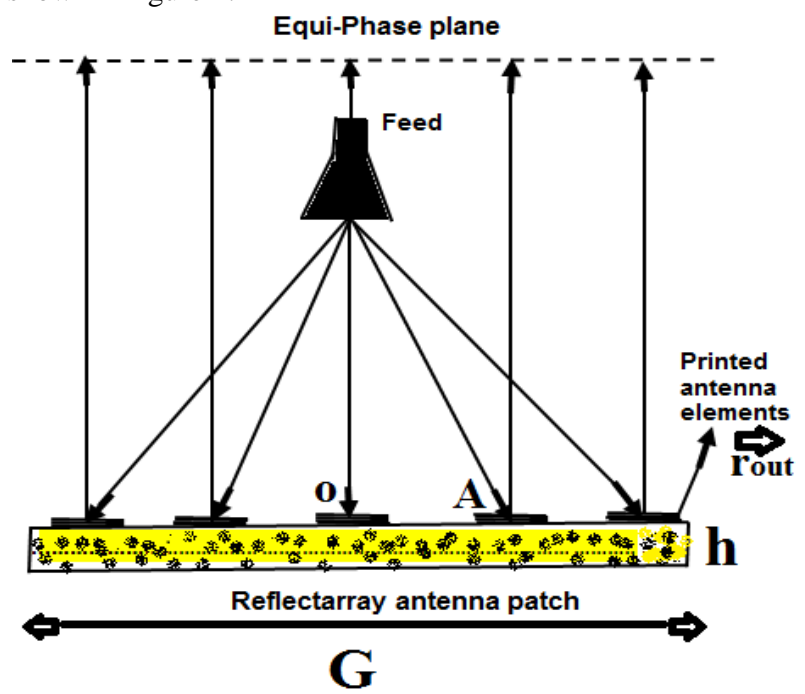

Fig 2: Reflectarray Configurations

The feed horn antenna is shown as $\mathbf{F}$, the height of dielectric substrate is depicted as $\mathbf{h}$, the ground plane at the bottom of the substrate can be shown as $\mathbf{G}$, the incident fields are shown as $\mathbf{O}$ and $\mathbf{A}$ and the reflected field is depicted as rout. The arrangement of elements is considered as the crucial part in the design of reflectarray antennas. The performance of reflectarray antennas is totally depended on the selection of suitable unit cell elements. The cell elements are then placed in a specific geometric location to get the desired results of phase transformation. Estimation of reflectarrays by an infinite periodic structure leads to the smooth variation of the geometrical characteristics of the elements of the cell.

A classical Reflectarray cellular element with Lstub turned into used on the preliminary phases of researches as shown in the Figure 3. Here the stub is meant to be a small strip transmission line; the incident filed on the patch is re-emitted with a phase shift as measured by the length of the stub's patch [4]. The reflectarrays made up of these types of elements fallouts in relatively narrow bandwidth and high cross polarization.

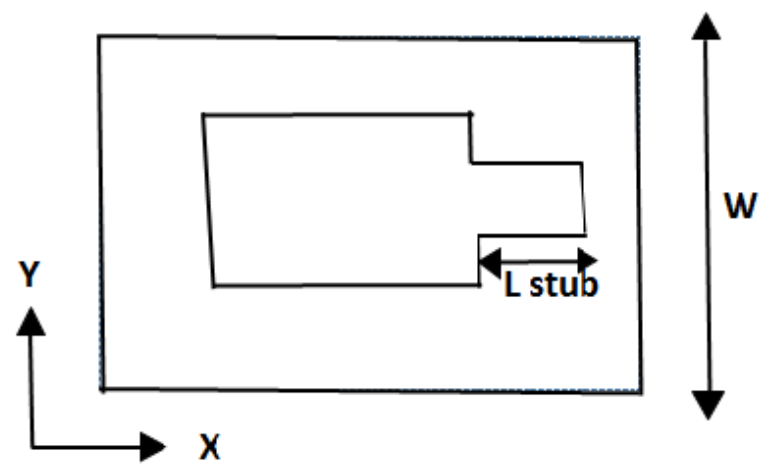

Fig 3: Reflectarray cell element with Lstub

The illusory radiations which are generated by the stub element can then change the required phase and also lower the level of cross polarization. The most conjoint kind of cell element is the "Rectangular Patch". Latterly inventions proved that patch elements can also be available on different sizes and shapes. Thus, linear polarization is achieved by using rectangular or square patches elements and dual or circular polarization is achieved by using circular patches or cross dipoles. These variable layout patches are commonly desired over the stub loaded- patch factors by means of retaining constant width and changing length of those patches as depicted in underneath Figure 4.

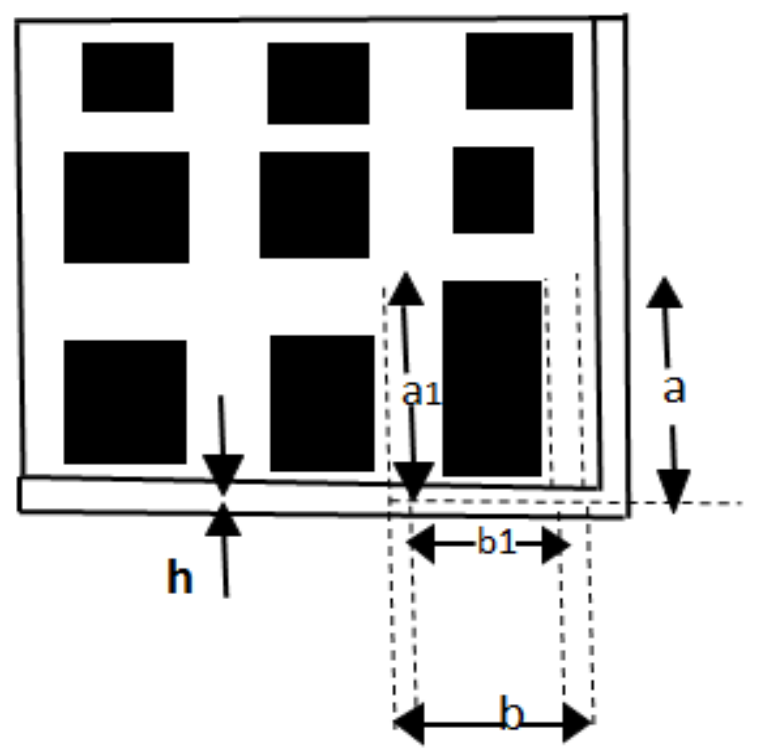

Fig 4: Variable size patch elements of Reflectarray 
The required-phase ( $\varphi$-patch) that could to be contributed through the square patch can be measured by means of using equation-1.

$$
(\text { FA }- \text { FO.rout })-\varphi p a t c h=2 \pi N
$$

Table 1. Comparison of Reflectarray with other Reflector antennas

\begin{tabular}{|l|l|l|l|}
\hline $\begin{array}{l}\text { Performance } \\
\text { Parameter }\end{array}$ & Reflectarray & $\begin{array}{l}\text { Parabolic } \\
\text { Reflector }\end{array}$ & $\begin{array}{l}\text { Phased } \\
\text { Array }\end{array}$ \\
\hline Gain & High & High & High \\
\hline Bandwidth & Low/Moderate & High & Moderate \\
\hline $\begin{array}{l}\text { Beam } \\
\text { Steering }\end{array}$ & Electronic & Mechanical & Electronic \\
\hline Cost & Low & Moderate & High \\
\hline $\begin{array}{l}\text { Design } \\
\text { Complexity }\end{array}$ & Low & High & High \\
\hline $\begin{array}{l}\text { Structure } \\
\text { Low Weight, }\end{array}$ & $\begin{array}{l}\text { Bulky, } \\
\text { Paraboloid }\end{array}$ & $\begin{array}{l}\text { Moderate, } \\
\text { Planar }\end{array}$ \\
\hline
\end{tabular}

\section{REFLECTARRAY ANTENNA BANDWIDTH}

Bandwidth of reflectarray antenna is calculated by shifting $10 \%$ or $20 \%$ above the most loss value as shown in figure 5 .

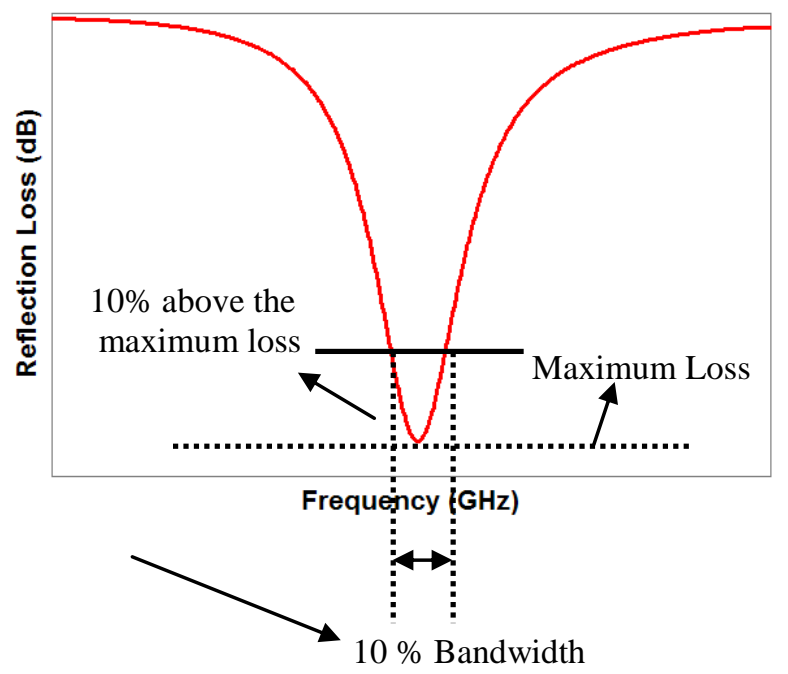

Fig 5: Bandwidth of Reflectarray Antenna

\section{CST MODELING AND SIMULATIONS}

Different styles of simulations have been carried out for the evaluation of reflectarray elements based on Finite Integral Method (FIM) [5]-[6] used in commercially available computer model of CST - Microwave Studio ${ }^{\circledR}$ (CST MWS). This method was initially proposed by Thomas Weiland in 1977, which has been improved incessantly over the years. The simple concept on this technique is to use the Maxwell's equations in integral form to a set of staggered grids. Infinite reflectarrays have been designed using proper boundary conditions. Different configurations and materials have been used for the study of performance of reflectarrays. After understanding the fundamentals of reflectarrays the second step is to carry out the primary evaluation to analyze the various factors which can have an effect on the performance of reflectarrays. Some of the factors together with the consequences of material properties, results of substrate thickness and outcomes of various element configurations have been considered in this evaluation.

\section{MEASUREMENT SETUP}

The fabricated samples are then required to be measured by the network analyzer using waveguide simulator technique. The measurement setup requires a coax to waveguide adapter to connect the waveguide with the analyzer [7]. The incident energy will be launched by the network analyzer to the waveguide via this connector which is required to offer the lowest reflection coefficient and most repeatable measurement, as compared to all other connectors. This is the preferred connector for most of the applications where least losses due to interconnections are tolerable and accuracy is considered critical. The two-patch detail reflectarray unit cell can be measured by way of putting into the aperture of the waveguide as shown in figure 6 . Reflection loss and reflection phase overall performance of different fabricated elements can be easily realized by means of the scattering parameter measurements [6].

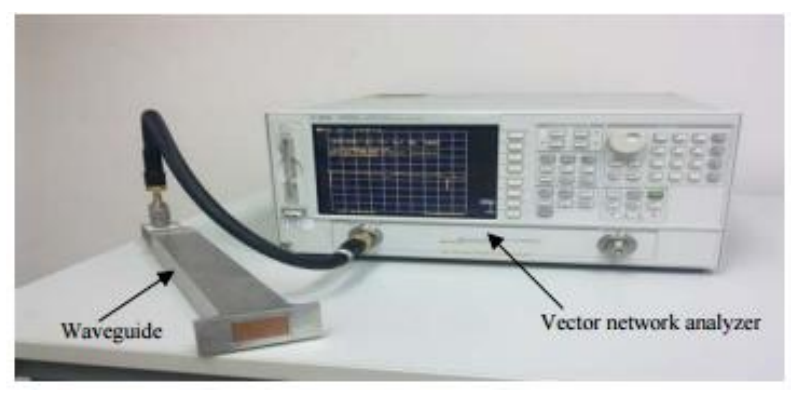

Fig 6: Scattering parameter measurements setup for two patch unit cell reflectarrays

\subsection{Waveguide Simulator and Unit Cell Reflectarray}

Since $19^{\text {th }}$ century, the waveguides are used as the transmission lines for the propagation of electromagnetic signals. The general structure consists of a hollow metallic tube with the conducting walls surrounded by the free space. The overall structure might be bulky; however they are highly suitable for the power handling and loss performance applications. In case of arrays, the effect of surroundingelements is necessary to be kept into account for the precise characterization of the unit cell element [5]. The waveguides comes in handy as they help to realize the unit cell as the infinite arrays. In order to bring out the scattering parameter measurement of infinite reflectarrays, a waveguide is required to be fabricated [8]. Consider a rectangular waveguide; it can propagate with distinct modes include the Transverse Electric (TE) and Transverse Magnetic (TM). These modes have a cut off frequency under which propagation isn't always feasible. Rectangular waveguide is suitable for this purpose because of ease of fabrication and ability of both T.E and T.M mode. The cut-off frequency of the rectangular waveguide is given by way of the subsequent equation [9].

$$
f_{\text {Cmax }}=\frac{c}{2 \pi} \sqrt{\left(\frac{m \pi}{a}\right)^{2}+\left(\frac{n \pi}{b}\right)^{2}}
$$

Where, $\mathrm{c}$ is considered as the light speed, $\mathrm{a}$ and $\mathrm{b}$ are considered as the broader side widths and length of the rectangular waveguide respectively. For the scattering 
parameter measurements, the two patch unit cell reflectarrays are required to be fabricated on the selected material (Rogers 5880). The design of the two patch unit cell reflectarrays is presented in Figure 7.

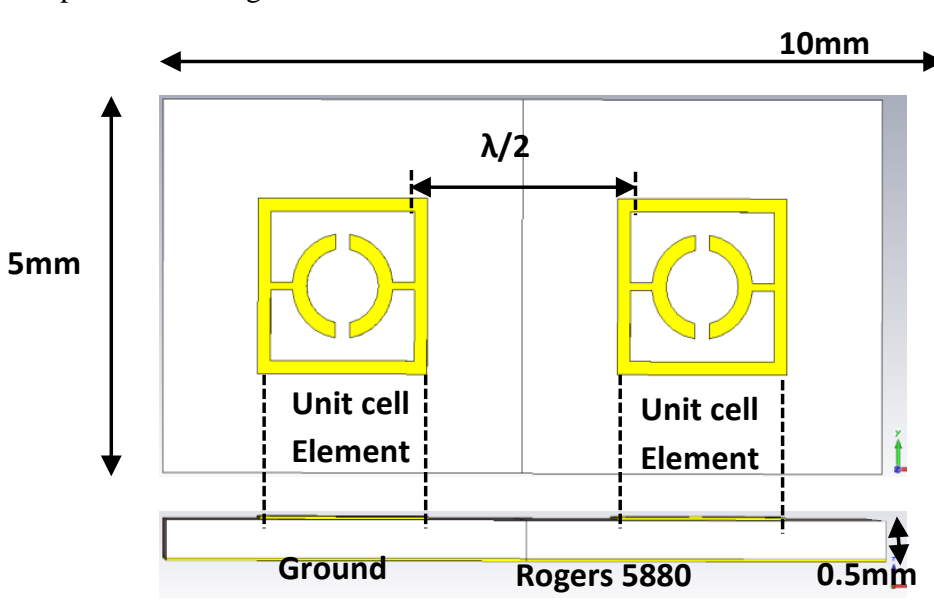

Fig 7: Two patch unit cell reflectarray for scattering parameter measurements

The thickness of the substrate is $0.5 \mathrm{~mm}$ and the inter-element spacing between the two elements is half wavelength $(\lambda / 2)$. This spacing is recommended in arrays to keep away from the arising of grating lobes and to keep away from the viable mutual coupling effects among the adjacent elements. Once the design is finalized, it is able to be fabricated by using the printed circuit technology making use of photo etching process.

\section{PERFORMANCE PARAMETERS}

\subsection{Static Phase Range and Figure of Merit (FOM}

In order to lessen the phase errors better static phase range is required. Figure of Merit (FoM) has been defined as "the ratio of the change in reflection phase to the change in the frequency".

$$
\mathrm{FOM}=\frac{\Delta \emptyset}{\Delta f}(\% M H z)
$$

The reflection phase in degrees is denoted as $\Delta \varphi$, and the change in the resonant frequency in $\mathrm{MHz}$ of the reflectarray antenna is denoted as $\Delta \mathrm{f}$. It has been located that the FOM increases, with the growth in dielectric permittivity. This is for the cause that growing the permittivity, causes the reflection phase to get steeper and therefore $\Delta \varphi$ reasons to growth over the equal collection of frequencies. Figure of Merit (FoM) describes the slope of phase-curve [10]. Higher the FOM value, the slope of phase curve will be maximum. FOM estimates the bandwidth performance; in terms of reflection phase-curve. For the comparison, Table 2 shows the static phase range and FoM values of both unit cell reflectarrays. From Table 2 it has been found that circular loop with the minimum static phase range of $95^{\circ}$ gives a minimal FOM value of $0.11^{\circ} / \mathrm{MHz}$ [11]. Furthermore, the square loop offers a maximum static phase range of $100^{\circ}$ with a maximum FoM $0.86 \% \mathrm{MHz}$ [12]. All the performance parameters are presented and compared in detail in below Table 2, accordingly.
Table 2: Performance comparison of unit cell reflectarrays for $5 \mathrm{G}$ communications

\begin{tabular}{|c|c|c|}
\hline $\begin{array}{c}\text { Unit-Cell } \\
\text { Reflectarray } \\
\text { Element Type }\end{array}$ & $\begin{array}{c}\text { Circular Loop } \\
\text { with Split Rings }\end{array}$ & $\begin{array}{c}\text { Square Loop } \\
\text { with Split Rings }\end{array}$ \\
\hline $\begin{array}{c}\text { Resonant } \\
\text { Frequency (GHz) }\end{array}$ & 28 & 28 \\
\hline $\begin{array}{c}\text { Surface Current } \\
\text { Density (A/m) }\end{array}$ & 2062 & 2472 \\
\hline $\begin{array}{c}\text { Reflection Loss } \\
(\text { dB) }\end{array}$ & 0.65 & 0.8 \\
\hline $\begin{array}{c}\text { 10\% Bandwidth } \\
\text { (MHz) }\end{array}$ & 60 & 52.4 \\
\hline $\begin{array}{c}\text { Static Phase } \\
\text { Range (Degrees) }\end{array}$ & 05 & 100 \\
\hline $\begin{array}{c}\text { Figure of Merit } \\
\text { (FoM) } \\
\text { (Degree/MHz) }\end{array}$ & 0.11 & 0.86 \\
\hline
\end{tabular}

\subsection{Reflection Loss and 10\% Bandwidth}

The reflection loss and reflection phases are the two most important factors to characterize the performance of unit cell reflectarrays designed and presented in the above section. Figure 8 (a.) and Figure 8 (b.) shows the reflection loss and reflection phase curves for the circular-loop with circular split rings and square-loop with circular split rings respectively [6]. It should be noted that both the reflectarray elements were designed and analyzed on the Rogers 5880 substrate with the thickness of $0.5 \mathrm{~mm}$. All parameters were kept constant in both the designs. It has been shown that the circular loop with circular split rings achieves the minimum surface current density offering the lowest reflection loss of $0.65 \mathrm{~dB}$ [8]. On the other hand, the square loop with circular split rings is demonstrated to achieve the highest surface current density depicting the highest reflection loss of $0.8 \mathrm{~dB}$. The reflection loss curves can provide us the bandwidth. The $10 \%$ bandwidth of unit cell reflectarrays can be obtained from its reflection loss curve by way of transferring $10 \%$ above the maximum loss value. Table 2 gives the $10 \%$ bandwidth values for each element beneath attention. It has been shown that the circular loop with lowest reflection loss achieves the highest bandwidth of $60 \mathrm{MHz}$. While, the square loop undergoes the highest reflection loss with the minimum bandwidth of $52.4 \mathrm{MHz}$. 


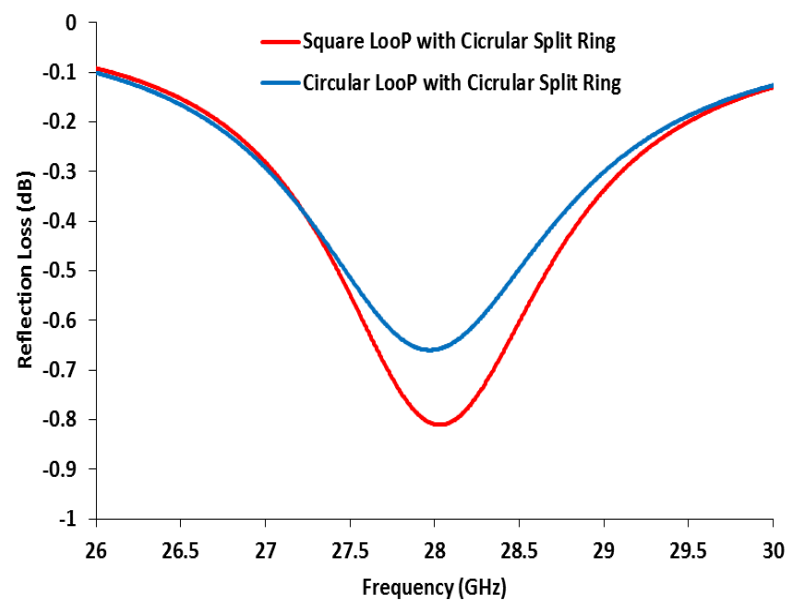

Fig 8 (a.)

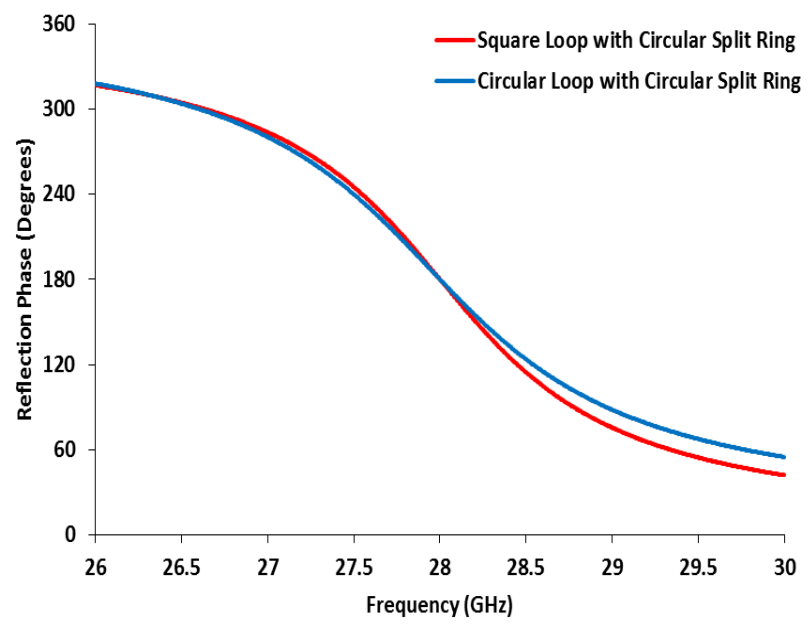

Fig 8 (b.)

Fig 8: Reflection loss and reflection phase performance for unit cell reflectarrays for $5 \mathrm{G}$ communications

\subsection{Reflection Phase and Static Phase Range}

Similarly, the reflection phase curves had been in comparison. The total phase range goes through the swing of $360^{\circ}$. Considering the reflection area of both the elements it could be seen that circular loop with split rings demonstrate the smoother phase slop as compared to the square loop with split rings, which achieves the steeper phase slope. The phase slopes can provide us the static phase range. The static phase range is the range at which the reflection phase shows the linearity in its variation. Additionally, the bandwidth of reflectarrays can also be measured by way of the slope of reflection phase versus curve of reflection frequency. The bandwidth of the reflectarrays may be lesser if the slope of the reflection phase curve is steeper [13]. Hence, there exist a change-off among the static phase range and the bandwidth.

\section{DESIGN CONFIGURATION, ANALYSIS AND RESULTS}

Here the effect of the port distance, variable substrate thickness and different permittivity's of the substrate materials were thoroughly analyzed before designing the reflectarrays for $5 \mathrm{G}$ communications.

\subsection{Effect of Variable Port distance}

Reflectarray is generally excited by a port at the distance of $\lambda_{\mathrm{g}}$ from the resonant element on top of the substrate. In this case, the port distance of the square patch reflectarray is varied from $2.5 \mathrm{~mm}$ to $7.5 \mathrm{~mm}$ in mandate to investigate the effect on reflection loss and reflection phase properties. The basic reason is to study the scattering behavior with the change in port distance. Initially, an infinite reflectarray was designed with square patch was to resonate at the central frequency of $28 \mathrm{GHz}$. The results are given below. Figure 9.0 (a) displays the reflection loss versus resonant frequency corresponding to different port distances. It can be seen that the element with the port distance of $2.5 \mathrm{~mm}$ demonstrate the reflection loss of $0.176 \mathrm{~dB}$. As the port distance is increased to 4.5 the reflection loss decrease to $0.172 \mathrm{~dB}$ and increase again with the increase in port distance to $7.5 \mathrm{~mm}$. This shows that the change in port distance does not affect the reflection loss value highly. There is just a slight variation in the overall reflection loss values.

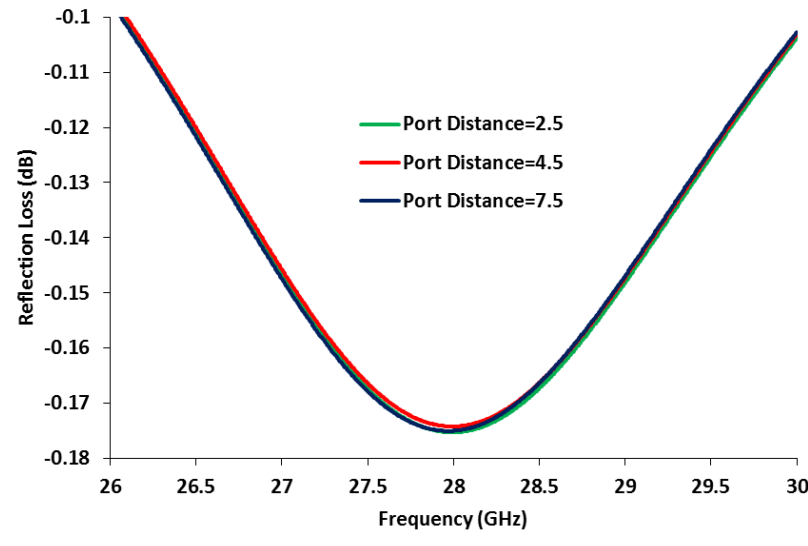

Fig 9 (a.): Reflection loss performance by varying port distance

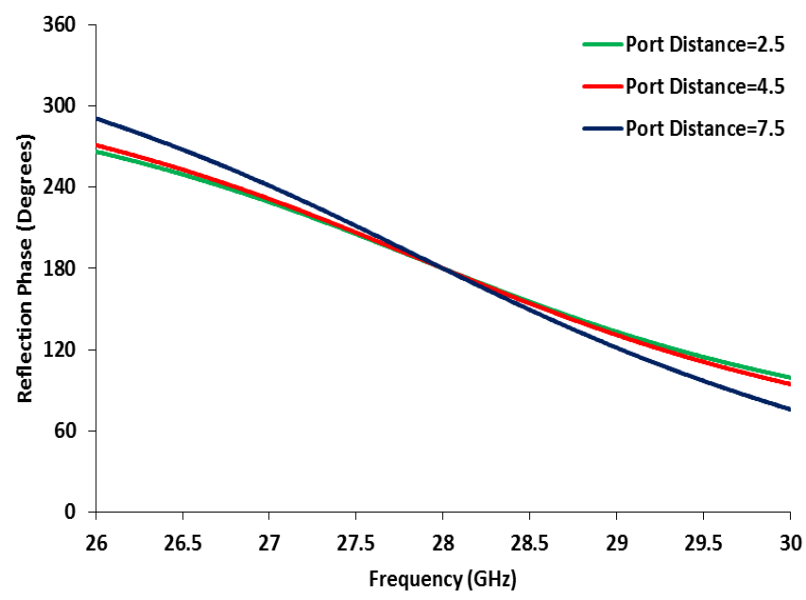

Fig 9 (b.): Reflection phase performance by varying port distance

Similarly, the reflection phase performance has been compared in Figure 9.0 (b). The trend shows that the element with port distance of $2.5 \mathrm{~mm}$ has smoother phase range as compared to the port distance of $7.5 \mathrm{~mm}$. It can be seen that the reflectarray element with the port distance of $7.5 \mathrm{~mm}$ undergoes the steeper phase range. However, the overall variation does not affect the refection phase performance. 


\subsection{Effect of Variable Substrate Thickness}

The substrate thickness additionally performs a critical position inside the evaluation and overall performance of reflectarrays. Square patch of the reflectarray was again utilized to investigate the effect of variable substrate thickness. In this example the substrate thickness became various from $0.5 \mathrm{~mm}$ to $1 \mathrm{~mm}$ by using preserving the constant loss tangent of 0.0009 . The reflection loss and reflection phase curves with variable substrate thickness are proven in Figure 10.0 (a) and Figure 10.0 (b) respectively [14]-[15]. It can be observed from Figure 10.0 (a) that the reflection loss decreases from $0.18 \mathrm{~dB}$ to $0.05 \mathrm{~dB}$ as the substrate thickness increased from $0.5 \mathrm{~mm}$ to $1 \mathrm{~mm}$. The resonance at the thinner substrate is better than the resonance at the thicker substrates. This is due to the fact that thicker substrates have concentration of incident energy. It has also been found that the reflection loss decreases with the boom within the substrate thickness [16]. It can be attributed due to the fact that the increase in substrate thickness reduces the multiple bounces within the substrate region.

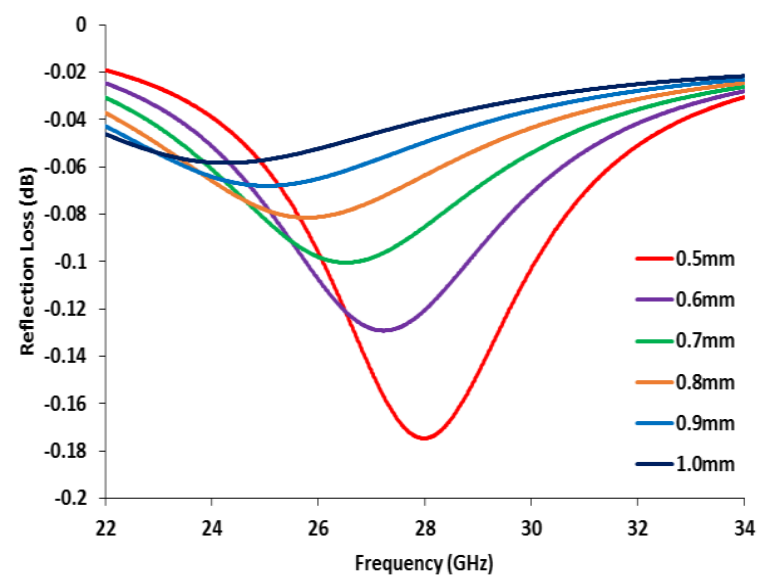

Fig 10.0 (a.): Reflection loss performance by varying substrate thickness

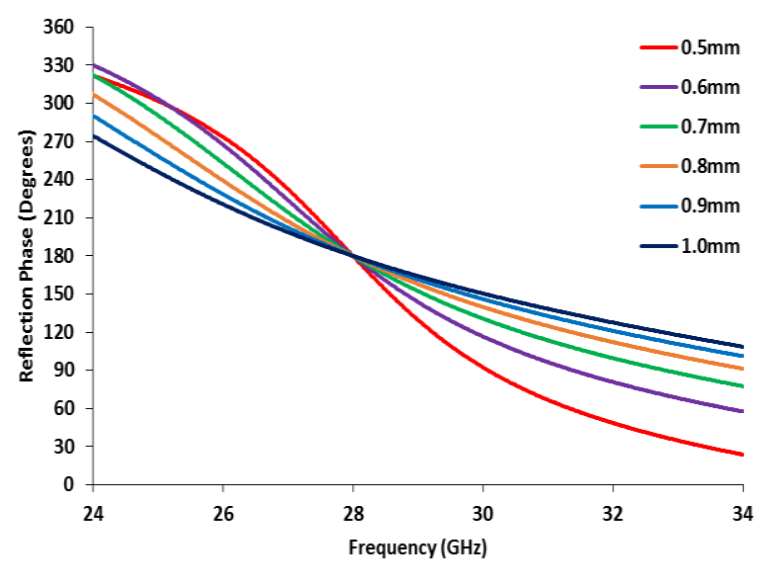

Fig 10.0 (b.): Reflection phase performance by varying substrate thickness

\subsection{Effect of Variable Substrate Permittivity}

Previous study provided an overview of the variable substrate thickness with fixed permittivity of the selected material. However, the change in permittivity is also required to be studied for the performance comparison of reflectarrays. In this study, initially the substrate permittivity was set to 2.2. The reflection loss and reflection phase performance was then observed by varying the permittivity up to 9.2. Other permittivity values that were considered include $3,4.5$ and 6.15 respectively. As shown in Figure 11.0 (a), the low dielectric permittivity of 2.2 results in very little absorption of energy in the substrate, which exhibits very low reflection loss of $0.1 \mathrm{~dB}$. On the other hand, the material with the very best permittivity of 9.2 validated higher reflection loss value of 0.9 $\mathrm{dB}$. It can be located that the bottom value of dielectric permittivity gives the bottom reflection loss and the best value of permittivity gives the highest reflection loss [15] as the values were varied from 2.2 to 9.2. The performance of variable substrate permittivity was also considered for the reflection phase performance as shown in the Figure 11.0 (b). Comparing the phase curves for each value of the substrate permittivity depicted the trend of the phase slope. The material with low permittivity of 2.2 exhibit smoother reflection phase. As the permittivity was increased the phase curve tends to become steep and it's steeper at the highest permittivity of 9.2. The slope of these phase curves also provide an effective way of measuring the bandwidth. However, increasing the phase range improves the bandwidth by trading off the bandwidth.

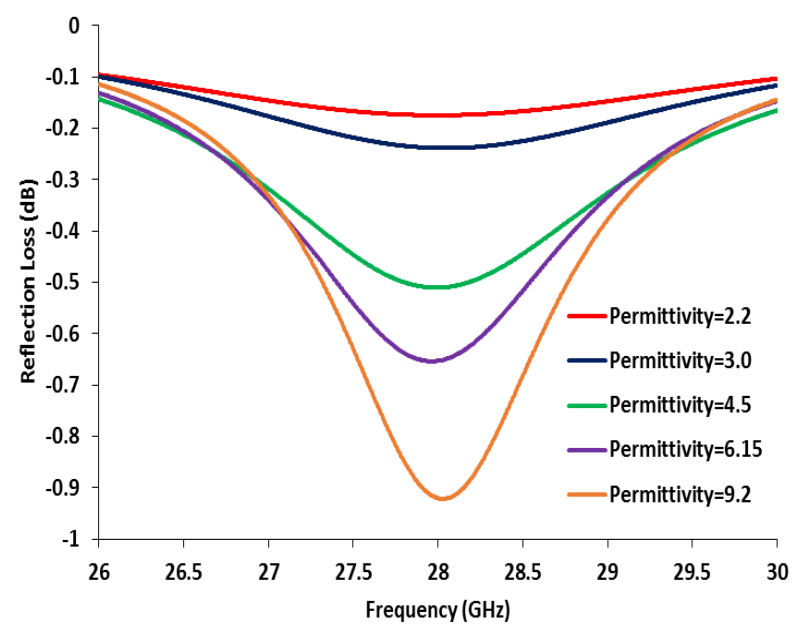

Fig 11.0 (a.): Reflection loss performance by varying substrate permittivity

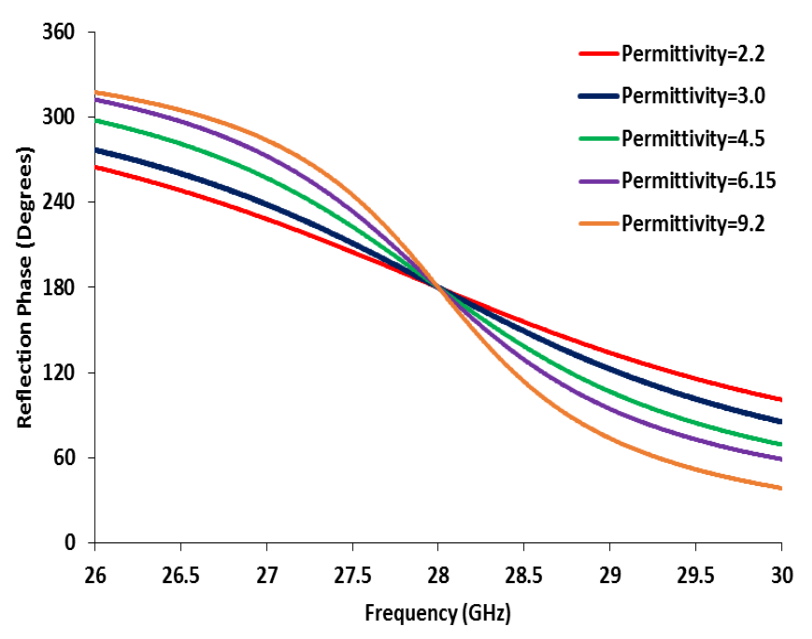

Fig 11.0 (b.): Reflection phase performance by varying substrate permittivity 


\section{SURFACE CURRENT DISTRIBUTION}

In this section, we compared the surface current on top of each element to understand the electrical behavior as demonstrated in Figure 12.0 (a) and Figure 12.0 (b). It is vital to note that the electric field is excited inside the Y-direction [9]. It can be seen that the maximum current is on the circular and square loops with minimum amount of current on the inner circular split rings. As depicted in Figure 12.0, the maximum current on the circular loop can be modified with square loop. The circular loop with split rings has surface current density that is proven to have at $2062 \mathrm{~A} / \mathrm{m} \mathrm{[10].} \mathrm{The}$ surface current density is further expanded up to some extent i.e. $2472 \mathrm{~A} / \mathrm{m}$ when the square loop with circular split rings is made to resonate at the same frequency. The circular loop occupies more area as compare to the square loop. Therefore the reduction in area results in higher current density. To further understand this, consider the element in terms of reflecting the incident energy. The element with larger area reflects back most of the incident energy offering low surface current density. On the alternative hand, in case of the element with smaller place, most of the incident energy relates with the substrate place depicting better surface - current- density [16]. This suggests that the surface current density on the elevated position of reflectarray element is inversely proportional to the reflection area of the element. A change in the surface current density can also cause a vast change in the reflection-loss and reflection-phase overall performance [5]. Thus, it can be determined that the change in the shape and reduction of reflectarray element area tends to achieve the higher surface current distribution as specified by the Maxwell's equation [10].

$$
I=\oint J \cdot d s
$$

$$
\text { Where, } J=\sigma E
$$

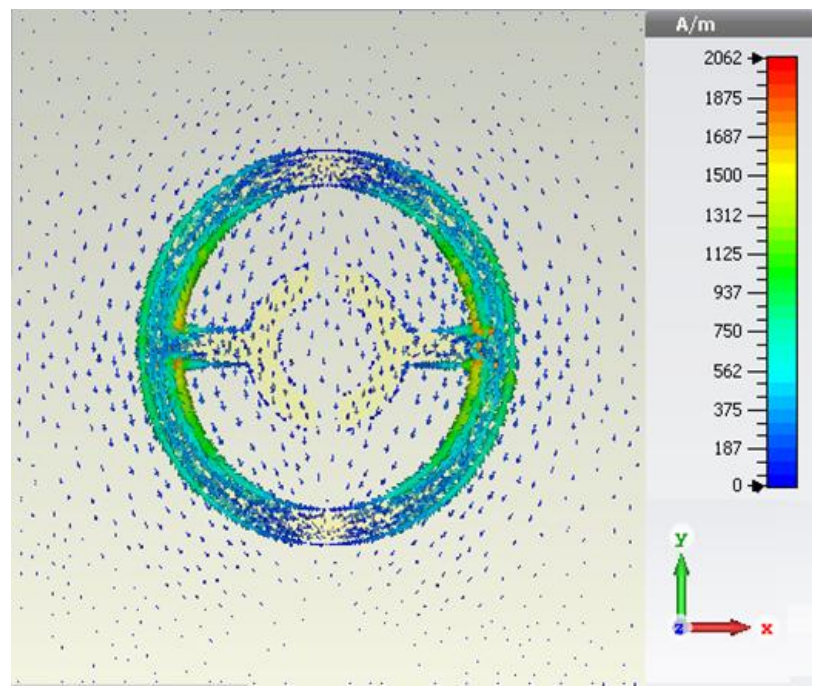

Fig 12.0 (a.): Surface Current Density on Reflectarray elements - Circular loop with circular split rings

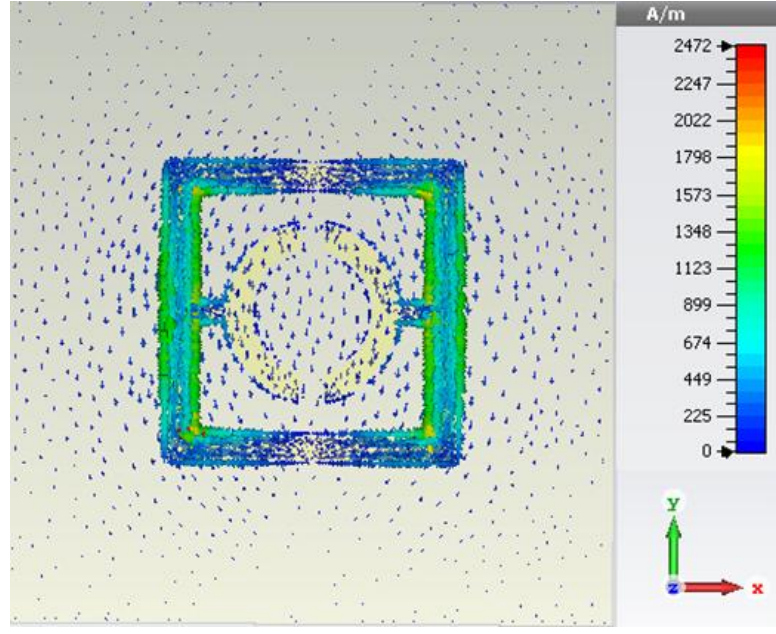

Fig 12.0 (b.): Surface Current Density on Reflectarray elements - Square loop with circular split rings

\section{CONCLUSION}

The rise of 5th generation mobile communication systems has witnessed increasing penetrations of modern smart phones and explosive growth of the data traffic networks. On one hand the attractive new services and applications demands higher transmission, higher data rate and adequate bandwidth. On the other hand, this rapid development requires planar, high-gain and cost effective antennas for better efficiency and lower network latency. Previously, bulky reflectors and expensive phased arrays were used to serve for the high-gain applications. However, reflectarray antennas being the hybrid design of both are planar, have low-weight and low cost. This thesis takes into account different design considerations to demonstrate the working and feasibility of reflectarrays as the potential antennas for the $5 \mathrm{G}$ applications. The performance of reflectarray antennas based on different unit cell resonant elements has been thoroughly investigated and presented in this work. The design and analysis has been carried out with in $\mathrm{Ka}$ band, specifically at $28 \mathrm{GHz}$ to target the $5 \mathrm{G}$ wireless communication applications. Two unique shapes of the reflectarray resonant elements were exploited in this thesis. These resonant elements had been printed on top of the dielectric substrate and analyzed in terms of reflection loss, reflection phase, $10 \%$ bandwidth and the static phase range. Commercially available CST MWS simulation tool has been utilized throughout the design and investigation stages. The results obtained by way of the Finite Integral Method (FIM) demonstrate that the required reflection performance can be executed via optimizing numerous layout parameters inclusive of port distance, substrate material and the substrate permittivity. It has been realized that for higher frequency i.e. $28 \mathrm{GHz}$, the port distance has no significant impact on the reflection properties of the reflectarray elements. However, it has been demonstrated that the change in substrate thickness or substrate permittivity can alter the reflection response accordingly. The higher the thickness of the substrate the lower is the $10 \%$ bandwidht and static phase range. Conversely, the better the permittivity of the substrate the higher is the $10 \%$ bandwidth and static phase range. It can be determined that there is a direct relationship between bandwidth and static phase range with the substrate thickness and an indirect relationship between bandwidth and static phase range with the substrate - permittivity. Next, two different unit cell reflectarray were designed based on the circular loop with split rings and square loop with split rings respectively. Reflection characteristics of the designed 
elements were thoroughly studied using the surface current density. It might be observed that that different unit cell element shape corresponds to a different reflection are which in turns change the over current distribution. The change in the current distribution can be studied in terms of reflection loss and reflection phase, and it can be extracted as the $10 \%$ bandwidth and the static phase range respectively. It has been observed that the circular loop with circular split rings achieves the minimum surface current density offering the lowest reflection loss of $0.65 \mathrm{~dB}$. Alternatively, the square loop with circular split rings is demonstrated to gain the highest surface current density depicting the highest reflection loss of $0.8 \mathrm{~dB}$. Also, the circular loops with split rings demonstrate the smoother phase slop as compared to the square loop with split rings, which achieves the steeper phase slope. Moreover, it has been determined that circular loop with the minimum static phase range of $95^{\circ}$ offers a minimum FOM value of $0.11^{\circ} / \mathrm{MHz}$. Furthermore, the square loop offers a maximum static phase range of $100^{\circ}$ with a maximum FoM $0.86^{\circ} / \mathrm{MHz}$. The results demonstrated the successful working of the designed unit cell reflectarray elements at $28 \mathrm{GHz}$. The shape and area of the elements had been proven to be the crucial factor to obtain the desired reflection performance. Therefore, it can conclude that the presented reflectarray antennas with further optimization are capable of replacing the conventional bulky and heavy weight reflector antennas. Furthermore, different techniques can be applied to enhance the reflection performance for the future wireless applications.

\section{FUTURE WORKS}

In order for reflectarray antennas to be adopted rapidly for the existing and upcoming $5 \mathrm{G}$ wireless applications, following works can be done

- Numerical analysis can be carried out to obtain the progressive phase distribution for larger array elements.

- Ultra wideband reflectarray antennas can be designed to cover the lower frequency bands for mobile handset MIMO terminals.

- Multi-band reflectarrays can be realized with the beam steering techniques to combat the scan blindness problem in various wirelessly controlled environments.

- Various techniques can be implemented for electronic switching such as design of unit cell reflectarrays with pin diodes and varactor diodes to achieve the dynamic phase range.

- Scattering parameter measurements can be accomplished the use of the waveguide simulator technique for unit cell reflectarrays.

- Chamber measurements can be taken for the design of a complete reflectarray based on the required number of array elements corresponding to the targeted application.

\section{ACKNOWLEDGMENTS}

With immense pleasure articulating propounded regards to vulnerable and highly esteemed guide Dr. Shahzada Alamgir Khan who guided with his valuable, experienced and technical knowledge in research and development as well. Special thanks to Dr. Irfan Zafar and my pen friend Mr. Arslan Kiyani who always willing to help and give their best suggestions during the entire period of MS degree and paper writing.

\section{REFERENCES}

[1] Abdul Samad, Kashif Azim and Shahzada Alamgir Khan Article: Comparative Analysis of Plasma Antenna with Metal Antenna. International Journal of Computer Applications 128(6):13-18, October 2015.

[2] "Handbook of Antenna Technologies", Springer Nature, 2016

[3] Han, Shuangfeng, Chih-lin I, Zhikun Xu, and Corbett Rowell. "Large-scale antenna systems with hybrid analog and digital beamforming for millimeter wave 5G", IEEE Communications Magazine, 2015.

[4] Qotolo, Sajjad Faraji, Hamid Reza Hassani, and Mohammad Naser-Moghadasi. "A novel broadband reflectarray antenna with lattice stubs on square element for Ku-band application", Microwave and Optical Technology Letters, 2015.

[5] M. Y. Ismail and Arslan Kiyani. "Investigation of reflection area on strategic reflectarray resonant elements", 2013 IEEE Symposium on Wireless Technology \& Applications (ISWTA), 2013.

[6] M. Y. Ismail, M. Inam, and N. Misran. "Characterization of resonant elements for passive and reconfigurable reflectarray design", 2014 5th International Conference on Intelligent and Advanced Systems (ICIAS), 2014.

[7] Theodore S. Rappaport et al., " Millimeter Wave Mobile Communications for 5G Cellular: It Will Work!' Millimeter Wave Mobile Communications, 2013.

[8] Inam, M., and M. Y. Ismail. "Integration of PIN diodes with slot embedded patch elements for active reflectarray antenna design", 2012 International Symposium on Telecommunication Technologies, 2012.

[9] Engineering Electromagnetics, 2015.

[10] Arslan Kiyani and M. Y. Ismail. "Numerical model for phase distribution characterization of _reflectarray elements", 2012 International Symposium on Telecommunication Technologies, 2012.

[11] Sulaiman, N.H., and M.Y. Ismail. "Dual Frequency XBand Reflect Array Antenna using Dual Gap", Procedia Engineering, 2013.

[12] Arslan Kiyani and M.Y. Ismail. "Design and Analysis of High Performance Reflectarray Resonant Elements", Procedia Engineering, 2013.

[13] Muhammad Inam Abbasi. "Reflection loss and bandwidth performance of X-band infinite reflectarrays: Simulations and measurements", Microwave and Optical Technology Letters, 01/2011

[14] M. Inam, M. Y. Ismail, A F M Zain, and M. A. Mughal. "Analysis of reflectarrays printed above variable substrate thicknesses", 2010 IEEE Asia-Pacific Conference on Applied Electromagnetics (APACE), 2010.

[15] M. Y. Ismail and Arslan Kiyani, "Characterization of Printed Reflectarray Elements on Variable Substrate Thicknesses", International Journal of Electrical, Computer, Energetic, Electronic and Communication Engineering, 2014.

[16] Dahri, M. Hashim, and M.Y. Ismail. "Performance Analysis of Reflectarray Resonant Elements based on Dielectric Anisotropic Materials", Procedia Engineering, 2013. 